\title{
Recombination Line Abundances in the Winds of the [WC] Wolf-Rayet Stars
}

\author{
R. L. Kingsburgh ${ }^{1}$, I. Dashevsky ${ }^{1}$ and M. J. Barlow ${ }^{2}$ \\ ${ }^{1}$ York University, Dept. of Physics and Astronomy; \\ ${ }^{2}$ University College London, Dept. of Physics and Astronomy
}

\begin{abstract}
We present a preliminary abundance analysis, utilising recombination theory, for the [WC] Wolf-Rayet central stars of the planetary nebulae NGC 6751 and NGC 6905. This analysis is based on optical spectrophotometry of a sample of [WC] stars which show a strong $\mathrm{O}$ VI $3811,34 \AA$ feature in emission. We have performed a recombination line analysis of stellar wind emission lines which are judged to be optically thin, in order to derive relative $\mathrm{C}, \mathrm{O}$ and $\mathrm{He}$ abundances. We also present a comparison of the derived wind abundances with those of Population I WO stars. For the [WC 4] central star of NGC 6751, we have derived $\mathrm{C} / \mathrm{He}=0.35$ and $\mathrm{C} / \mathrm{O}=4.2$, by number. And for the [WC 3] central star of NGC 6905 , we have derived $\mathrm{C} / \mathrm{He}=0.36$ and $\mathrm{C} / \mathrm{O}<12$. These surface abundances are comparable to the abundances that have been derived for early type [WC] stars via more complex NLTE modelling (e.g. Koesterke \& Hamann 1996).
\end{abstract}

\section{Introduction}

The [WC] Wolf-Rayet stars are a hydrogen-deficient sub-group of the central stars of PN. Their spectra exhibit strong broad emission line features, indicative of stellar winds similar to those found in the Population I WC and WO stars. Of particular interest are the members of the O VI sequence defined by Smith \& Aller (1969) which show the O VI 3 p-3s doublet at $3811,34 \AA$ strongly in emission, since the spectra of these stars shows the highest degree of ionization amongst all WR stars. Their spectra are dominated by lines of He, C and O. Unlike the Pop. I WR stars, there are no [WN] counterparts to the [WC] central stars, although occasionally the N V $1240 \AA$ feature is seen, indicating that although both Pop. I and II stellar winds reveal He-processed material, different mixing histories would have been experienced by the very different progenitor stars, allowing for trace amounts of nitrogen to be present in the Pop. II [WC] stars.

We have initiated a study of the O VI-sequence [WC] stars, aimed at deriving global parameters including their surface abundances and radiative energy distributions, in order to obtain insight into their evolutionary status. The current work presents a preliminary analysis for NGC 6751 and NGC 6905.

\section{Observations}

Both narrow- and wide-slit spectrophotometry were obtained using the RGO Spectrograph at the $3.0 \mathrm{~m}$ AAT, with an IPCS as detector. Data reduction was performed as described by Kingsburgh \& Barlow (1994).

Equivalent widths, EW, were measured from the narrow-slit spectra, while the absolute level of the underlying continuum, $\mathrm{F}_{c}(\lambda)$, was determined from the wide-slit spectra. Multiplication of the EW's by the appropriate values of $F_{c}(\lambda)$ yielded absolute line fluxes. These were dereddened by adopting the extinction coefficient derived from the Balmer decrement of the nebular spectrum (Kingsburgh \& Barlow, 1994) together with the Galactic reddening law of Howarth (1983). 


\section{Central Stars}

\section{Recombination line analysis}

A Case B recombination line analysis was performed for lines which were judged to be optically thin. The method is described in detail by Kingsburgh, Barlow \& Storey (1995). The adopted wind temperature and density were $50000 \mathrm{~K}$ and $10^{11} \mathrm{~cm}^{-3}$ respectively. Relative carbon, oxygen and helium abundances were derived for NGC 6751 and NGC 6905, and are presented in Table 1, along with the abundances derived for the Population I WO stars by the same method.

The oxygen abundance derived for NGC 6905 is currently a lower limit, based only on the $\mathrm{O}^{6+} / \mathrm{He}^{2+}$ abundance, as the OV $5590 \AA$ line is predicted to be partially optically thick, and cannot be used to estimate the $\mathrm{O}^{5+}$ abundance. The accuracy of the oxygen abundances for both objects will improve as UV spectra are incorporated into the analysis. The $\mathrm{C} / \mathrm{He}$ and $\mathrm{C} / \mathrm{O}$ ratios for NGC 6751 and 6905 are within $30 \%$ of the values found for the two galactic population I WO stars, Sand 4 and Sand 5, although these stars have had completely different evolutionary histories; the pop I WC/WO stars originating from $\sim 40$ $60 \mathrm{M} \odot$ stars, and the Pop. II [WC] stars from $\sim 1 \mathrm{M} \odot$ stars.

NGC 6751 has recently been analyzed by Koesterke and Hamann (1996; KH) using a detailed non-LTE model of the stellar wind. They derive the following mass fractions: $\mathrm{X}(\mathrm{C})=0.31, \mathrm{X}(\mathrm{O})=0.15, \mathrm{X}(\mathrm{He})=0.54$. From Table 1 , we derive the following mass fractions for NGC 6751: $\mathrm{X}(\mathrm{C})=0.44, \mathrm{X}(\mathrm{O})=0.14, \mathrm{X}(\mathrm{He})=0.42$. Our $\mathrm{X}(\mathrm{O})$ is in agreement with that of $\mathrm{KH}$, however our $\mathrm{X}(\mathrm{C})$ is a factor of 1.4 higher. The C IV $5470 \AA$ line is best suited for deriving carbon abundances (Hillier 1989); however KH's models underestimate this line's strength, and they conclude that a value of $\mathrm{X}(\mathrm{C})$ as high as $\sim 0.5($ or $\mathrm{X}(\mathrm{C}) / \mathrm{X}(\mathrm{O})=1.0)$ cannot be ruled out.

Table 1. A Comparison Between Population I and II Wolf-Rayet Wind Abundances By Number

\begin{tabular}{lllrrrc}
\hline & Object & class & C/He & O/He & C/O & Ref \\
\hline pop I & NGC 6751 & [WC4] & 0.35 & 0.086 & 4.2 & 1 \\
& NGC 6905 & [WC3] & 0.36 & $>0.029$ & $<12$ & 1 \\
pop II & Sand 1 & WO4 & 0.81 & 0.30 & 2.7 & 2 \\
& Sand 2 & WO4 & 0.52 & 0.11 & 4.6 & 2 \\
& Sand 4 & WO1 & 0.51 & 0.11 & 4.6 & 2 \\
& Sand 5 & WO2 & 0.52 & 0.10 & 5.2 & 2 \\
\hline
\end{tabular}

Notes: 1-this work; 2- Kingsburgh, Barlow \& Storey 1995.

\section{REFERENCES}

Howarth, I. D., 1983, MNRAS, 203, 301.

Hillier, J. D., 1989, ApJ, 347, 392

Kingsburgh, R. L, \& Barlow, M. J., 1994, MNRAS.

Kingsburgh, R. L, Barlow, M. J. \& Storey, P. J., 1995, A\&A, 295, 75.

Koesterke, L. \& Hamann, W.-R., 1996, A\&A, in press. (KH)

Smith, L. \& Aller, L. H., 1969, ApJ, 157, 1245. 